interpreted as the invisible population of the forests. Indian uncontacted is a legal concept that defines human populations from pre-Columbian culture that have remained geographically and culturally distant from the western population. Its demographic composition are unknown, registering only some evidence of their existence and meagre or no evidence of their material culture, customs and languages (Azanha, Gilberto. FUNAI-Mimeo, 2006). The Indian Protection Service of National Indian Foundation-SPI/ FUNAI finds that between 1910 and 1957, of the 105 known isolates indians, 72 were contacted and one third of those contacted were extinct. It is estimated that currently about 33 indigenous peoples remain uncontacted. At the time, especially measles and epidemic influenza were the main causes of the extermination of tribes contacted, currently the diseases that most affect the indigenous population are malaria and hepatitis. (Ribeiro, Darcy, 1996). The study describes the current situation and discusses strategies for preventing and controlling diseases in areas inhabited by these Indians in Brazil. The method establishes the parameters of health risks and establishing a surveillance system for the actions of prevention and control of these risk factors to health in these areas.

\section{P1-521 IO IN CHILDHOOD AND LATER RISK OF ALCOHOL-RELATED DISEASE AND DEATH}

doi:10.1136/jech.2011.142976h.9

\section{S Sjölund, ${ }^{*}$ T Hemmingsson, P Allebeck. Karolinska Institutet, Stockholm, Sweden}

Introduction While much research has been performed on associations between intelligence and several different health outcomes, there is as yet no conclusive evidence regarding the association between intelligence and alcohol-related disease and death. Furthermore, most studies have been performed on men. The aim of this study is to further investigate the association between IO and alcohol-related morbidity and mortality and to explore if there are any differences between men and women in this regard.

Method 43980 Swedish subjects (men and women) born 1948, 1953, 1967, 1972 and 1977 who completed an IQ test at age 13 were followed until 2005. Information on alcohol-related disease and death (ICD-diagnoses) has been collected from the National Patient register and the National Cause of Death register, respectively. Adjustments are made for parental socioeconomic position, age, income, education and occupation, obtained from national censuses. ORs for alcohol related disease and death will be estimated using Cox regression models.

Results So far, unadjusted results have shown, in general, a graded reduction in rates of alcohol-related death and disease by increasing IO. This was found both for men and as for women. Results from the Cox regression will be presented, crude and adjusted for both men and women.

Conclusion This study will contribute to the research investigating the association between IQ and alcohol-related disease and death. It is of particular interest to analyse this association also in women.

\section{P1-522 CLUSTERING OF HEALTH BEHAVIOURS IN SCOTLAND: A COMPARISON BETWEEN EAST AND WEST SCOTLAND AND BETWEEN URBAN AND RURAL REGIONS}

doi:10.1136/jech.2011.142976h.10

J Smith, ${ }^{*}$ R Mitchell. University of Glasgow, Glasgow, UK

Introduction There is evidence that health behaviours cluster together in certain patterns and that this varies by individual and socio-demographic characteristics. This study took a different perspective by investigating whether the clustering of health behaviours varies geographically in Scotland.

Methods Secondary, cross-sectional data from the Scottish Health Survey (2003) were analysed. The prevalence of meeting national recommendations on four health behaviours-alcohol, smoking, physical activity and fruit and vegetable consumption-was calculated. Two geographical divisions were made-east and west, and urban and rural. The number of healthy behaviours individuals have was analysed geographically, using binary logistic regression to control for confounders. Clustering was investigated using observed to expected ratios for each pattern of health behaviour, a method which indicates which patterns occur more or less frequently than would be expected by chance.

Results Prevalence of healthy behaviour differed significantly across both geographical divisions, except for alcohol behaviour. After controlling for age, sex and deprivation, a significant but small difference in number of healthy behaviours was found between urban and rural areas, but not between east and west. The most prevalent pattern of behaviour was not drinking or smoking, but also not eating enough fruit and vegetables and not exercising enough. Clustering of health behaviours did not differ geographically.

Conclusion Clustering of health behaviour may not contribute to geographical health inequalities in Scotland. However, the occurrence of multiple unhealthy behaviours was common and may be an important consideration for public health programmes in Scotland.

\section{P1-523 SOCIOECONOMIC-RELATED INEQUALITY IN ORAL HEALTH RISK BEHAVIOURS AMONG ADOLESCENTS IN THAILAND}

doi:10.1136/jech.2011.142976h.11

T Somkotra. ${ }^{*}$ Chulalongkorn University, Bangkok, Thailand

Introduction As risk behaviours, including unhealthy diet and nutrition, tobacco and alcohol consumption, relevant to noncommunicable chronic diseases are common to oral disease. This study aimed to quantify the extent to which socioeconomic-related inequality in oral health risk behaviours among Thai adolescents is manifested.

Methods Data of 5819 and 4429 Thai adolescents aged 11-19 years living in urban and rural, respectively from the nationally representative Health and Welfare Survey 2009 were used. Descriptive analyses were performed to reveal the features of oral health risk behaviours comprising frequency of eating snacks, drinking sweetened beverages, smoking, alcohol drinking. In addition to the use of concentration index (CI) to quantify the extent of socioeconomicrelated inequalities in these behaviours.

Results Reports of eating snacks and drinking sweetened beverages often (ie, more than 5 days per week) of those adolescents of higher socioeconomic-status were more common than their lower socioeconomic-status counterparts. By contrast, smoking and alcohol consumption reveals reverse gradients as these behaviours were more concentrated among those of lower socioeconomic-status than their peers counterparts. The aforementioned findings are corroborated by $\mathrm{CI}$ values that revealed pro-rich inequality in diet behaviours meanwhile the CI indicates pro-poor inequality in smoking and alcohol consumption.

Conclusion This study demonstrated socioeconomic-related inequality in oral health risk behaviours among Thai adolescents is discernable along the entire spectrum of socioeconomic status. The study also indicates population subgroups that should receive consideration for improving oral health risk behaviours which may be enable for integrated approaches in general health promotion strategies. 